\title{
BMJ Global Health Early implementation of guidelines for managing young infants with possible serious bacterial infection in Bangladesh
}

\author{
Jennifer A Applegate (D) , ${ }^{1}$ Sabbir Ahmed, ${ }^{2}$ Marufa Aziz Khan, ${ }^{2}$ Sanjida Alam, ${ }^{2}$ \\ Nazmul Kabir, ${ }^{2}$ Munia Islam, ${ }^{2}$ Mamun Bhuiyan, ${ }^{2}$ Jahurul Islam, ${ }^{3}$ \\ Iftekhar Rashid (1D , ${ }^{4}$ Steve Wall, ${ }^{5}$ Joseph de Graft-Johnson, ${ }^{5}$ Abdullah H Baqui, ${ }^{1}$ \\ Joby George ${ }^{2}$
}

To cite: Applegate JA, Ahmed S, Khan MA, et al. Early implementation of guidelines for managing young infants with possible serious bacterial infection in Bangladesh. BMJ Global Health 2019;4:e001643. doi:10.1136/ bmjgh-2019-001643

Handling editor Seye Abimbola

- Additional material is published online only. To view please visit the journal online (http://dx.doi.org/10.1136/ bmjgh-2019-001643).

Received 13 April 2019 Revised 22 October 2019 Accepted 25 October 2019

Check for updates

(c) Author(s) (or their employer(s)) 2019. Re-use permitted under CC BY-NC. No commercial re-use. See rights and permissions. Published by BMJ.

For numbered affiliations see end of article.

Correspondence to Dr Abdullah H Baqui; abaqui@jhu.edu

\section{ABSTRACT}

Neonatal infections remain a leading cause of newborn deaths globally. In 2015, WHO issued guidelines for managing possible serious bacterial infection (PSBI) in young infants ( $0-59$ days) with simpler antibiotic regimens if hospital referral is not feasible. Bangladesh was one of the first countries to adapt WHO guidance into national guidelines for implementation in primary healthcare facilities. Early implementation was led by the Ministry of Health and Family Welfare (MOHFW) in 10 subdistricts of Bangladesh with support from USAID's MaMoni Health System Strengthening project. This mixed methods implementation research case study explores programme feasibility and acceptability through analysis of service delivery data from 4590 sick young infants over a 15-month period, qualitative interviews with providers and MOHFW managers and documentation by project staff Multistakeholder collaboration was key to ensuring facility readiness and feasibility of programme delivery. For the $514(11 \%)$ infants classified as PSBI, provider adherence to prereferral treatment and follow-up varied across infection subcategories. Many clinical severe infection cases for whom referral was not feasible received the recommended two doses of injectable gentamicin and follow-up, suggesting delivery of simplified antibiotic treatment is feasible. However, prereferral antibiotic treatment was low for infants whose families accepted hospital referral, which highlights the need for additional focus on managing these cases in training and supervision. Systems for tracking sick infants that accept hospital referral are needed, and followup of all PSBI cases requires strengthening to ensure sick infants receive the recommended treatment, to monitor outcomes and assess the effectiveness of the programme. Only $11.2 \%$ (95\% Cl 10.3 to 12.1$)$ of the expected PSBI cases sought care from the selected service delivery points in the programme period. However, increasing trends in utilisation suggest improved awareness and acceptability of services among families of young infants as the programme matured. Future programme activities should include interviews with caregivers to explore the complexities around referral feasibility and acceptability of simplified antibiotic treatment.

\section{Summary box}

- Treatment with injectable gentamicin was high for infants with clinical severe infection when referra was not feasible; however, prereferral antibiotic treatment was low for infants whose families accepted referral to the hospital, especially for critically ill infants.

- Systems for routinely tracking sick infants that accept referral are needed to ensure families reach the higher-level facility, receive appropriate treatment and to monitor outcomes, which will require coordination within the Ministry of Health and Family Welfare to strengthen referral linkages between primary health facilities at the union and subdistrict level, and integration of indicators in routine monitoring systems.

- Few young infants sought care for possible serious bacterial infection from the programme area primary health facilities, which providers attributed to caregivers' preference for care from the private sector and lack of awareness about services offered at primary health facilities.

- Future programme efforts should include interviews with caregivers to explore the complexities around referral feasibility and acceptability of simplified antibiotic treatment and incorporate periodic observation of service delivery-including data quality checks of provider registers-to better assess the quality of care provided at primary health facilities and effectiveness of simplified antibiotic treatment.

\section{INTRODUCTION}

Bangladesh observed an impressive decline in under-five mortality and achieved the fourth Millennium Development Goal for child survival. ${ }^{1}$ Deaths in neonates, however, have been declining at a lower rate than mortality in older children. As a result, the proportion of under-five deaths occurring in the neonatal period increased from $44 \%$ in 1990 to $62 \%$ 
in $2015 .{ }^{1}$ With a neonatal mortality rate of 28 per 1000 live births, the country experiences over 74000 annual neonatal deaths. Most of these deaths are due to three causes: prematurity, infections and intrapartum-related complications. ${ }^{1-3}$ Progress towards reducing neonatal mortality due to these three causes is needed for Bangladesh to achieve the Sustainable Development Goal target of 12 neonatal deaths per 1000 live births by $2030 .^{1}$

Neonatal infections, including sepsis, meningitis and pneumonia, remain a leading cause of newborn death in Bangladesh. A recent population-based cohort study in rural Bangladesh attributed $46 \%$ of all neonatal deaths to serious infections. ${ }^{4}$ The WHO recommends that young infants ( $0-59$ days) with signs of possible serious bacterial infection (PSBI) be referred to hospitals and treated with a 7-10-day course of a combination of two injectable antibiotics: gentamicin and either penicillin or ampicillin. ${ }^{5}$ However, in resource-limited settings, many young infants with PSBI do not receive the recommended inpatient treatment because hospital care may not be accessible, acceptable or affordable. ${ }^{5}$ Based on trial data from South Asia and sub-Saharan Africa, in 2015, WHO issued new guidelines for managing PSBI in young infants, when referral is not feasible for families, with simpler antibiotic regimens that include fewer doses of injectable antibiotics coupled with oral antibiotics delivered by care providers closer to the community. ${ }^{5-8}$ Findings from these studies also contributed to a new evidence-base of implementation strategies for safely delivering outpatient treatment to newborns in resource-constrained settings. ${ }^{8-13}$

Recognising the need to increase access to and coverage of newborn health services, particularly management of PSBI, the Government of Bangladesh developed and implemented the National Newborn Health Program as part of the 4th Health, Population and Nutrition Sector Program. ${ }^{14}$ Bangladesh was one of the first countries to adopt the WHO guidelines and adapt them into national guidelines as part of a Comprehensive Newborn Care Package of interventions targeting leading causes of neonatal death in the country. ${ }^{14-16}$ Effective implementation of the updated guidelines requires multistakeholder partnership and collaboration across the health system. ${ }^{17}$ Implementation research provides an opportunity to study how interventions work in real world conditions, aiming to bridge the gap between evidence and practice to accelerate development and equitable delivery of health services. ${ }^{11} 1718$

Targeted primary health facilities for implementation of Bangladesh's national guidelines are Union Health \& Family Welfare Centers (UH\&FWC (catchment 25 000 persons) $).{ }^{16}$ In the past, UH\&FWCs have been largely underused by communities and many were not fully functional due to staff shortages and insufficient equipment. ${ }^{19-21}$ Evaluations of the related Integrated Management of Childhood Illness (IMCI) strategy in Bangladesh identified that primary health facilities, including UH\&FWCs, may not be well-equipped or supported to deliver IMCI services with notable gaps in providers' adherence to guidelines, supportive supervision and drug supply to treat severe illness in infants and children. ${ }^{22-24}$ Previous studies have found combining multiple knowledge transfer strategies-including training, distribution of job aides and regular supportive supervision-can improve health worker performance and sustain quality of care in health facilities. ${ }^{23} 25-28$

Guided by principles of implementation research, the Saving Newborn Lives project of Save the Children Bangladesh, USAID's MaMoni Health System Strengthening project (MaMoni HSS) and other development partners, collaborated with Bangladesh's Ministry of Health and Family Welfare (MOHFW) to support the rollout of national guidelines for managing PSBI in young infants at UH\&FWCs in selected districts. ${ }^{16}$ Here, we discuss MaMoni HSS' support to the MOHFW-led implementation of the guidelines in 10 subdistricts of three districts in Bangladesh, focusing on the project's implementation strategies-informed by WHO's guidance and embedded in the local context ${ }^{12}{ }^{13}$-and assessment of feasibility and acceptability through mixed methods analysis of programme data (figure 1). The aim of this implementation research case study is to share early programme learnings and provide recommendations for strengthening the programme as it is scaled-up in Bangladesh.

\section{CONTEXT AND INTERVENTION}

Bangladesh's MOHFW maintains a three-tier system for delivering healthcare services and is managed by two directorates: Directorate General of Health Services (DGHS) in the Health Service Division and Directorate General of Family Planning (DGFP) in the Medical Education and Family Welfare Division. ${ }^{14}{ }^{1920}$ Public sector healthcare service delivery follows the IMCI protocol for management of sick children at the community-level and facility-level. Implementation of the PSBI management guidelines is done by trained paramedics (SubAssistant Community Medical Officers (SACMO) and supported by Family Welfare Visitors (FWV)) at the UH\&FWC. The SACMO has 3 years training on general healthcare, including child health, and the FWV has 18 months training on midwifery and contraceptive management. ${ }^{19} 20$ The Upazila Health Complex ((UHC); catchment $\sim 250000$ persons), a subdistrict level hospital providing inpatient care, serves as the primary referral facility for PSBI cases. If a case cannot be managed at the UHC, then the infant is referred to secondary-level or tertiary-level care. ${ }^{1619}$

Per the national guidelines, the SACMO is the designated provider to assess the infant following a decision algorithm and provide management accordingly. Infants with PSBI are classified into three subcategories based on severity of illness (figure 2) ${ }^{16}$ Critical illness (CI) cases require inpatient management; thus, hospital referral with prereferral antibiotics is the only option for these infants. Young infants classified as clinical severe infection 


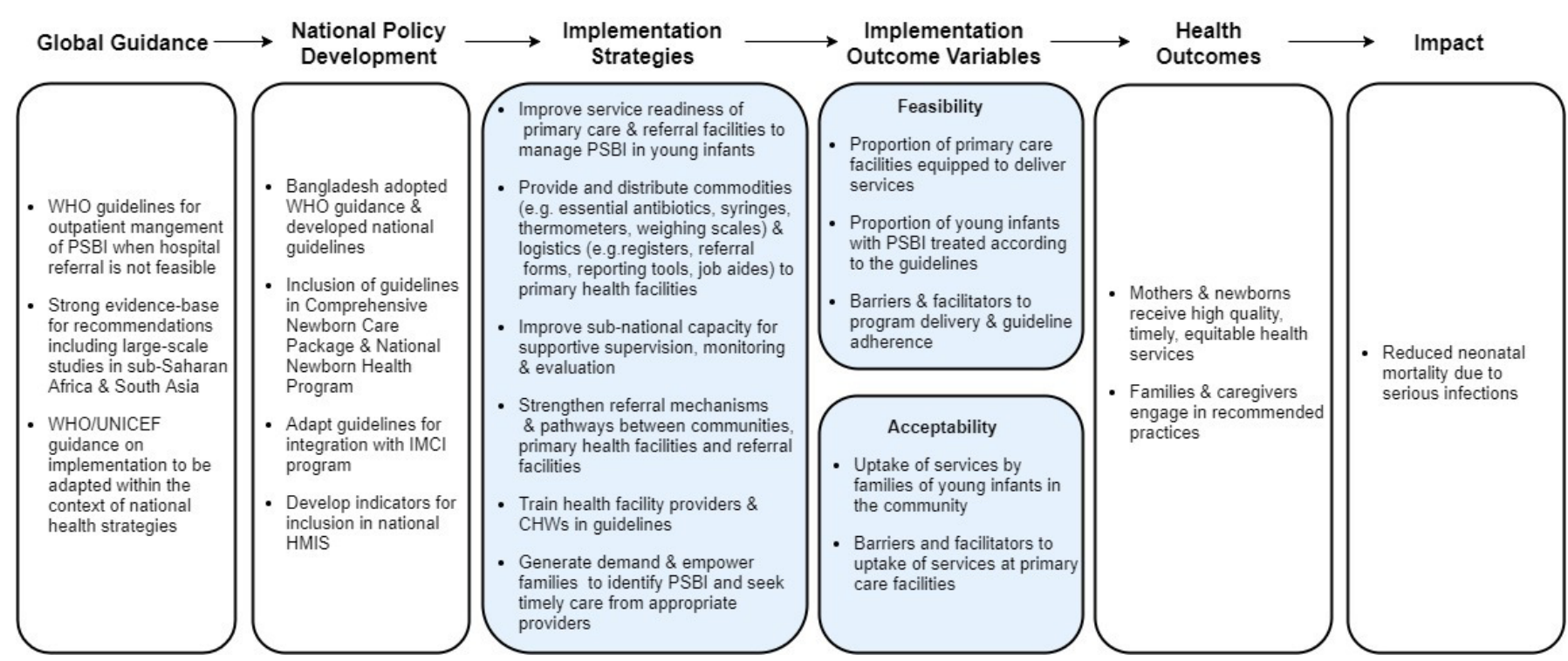

Figure 1 Conceptual framework for MaMoni HSS implementation research case study. Implementation strategies recommended by $\mathrm{WHO}$ and adapted to the local context. ${ }^{512}{ }^{13}$ Shaded boxes include the implementation strategies supported by MaMoni HSS and outcomes evaluated in our study. CHW, Community Health Worker; IMCl, Integrated Management of Childhood IIIness; HMIS, HealthManagement Information Systems; MaMoni HSS, MaMoni Health System Strengthening project; PSBI, possible serious bacterial infection.

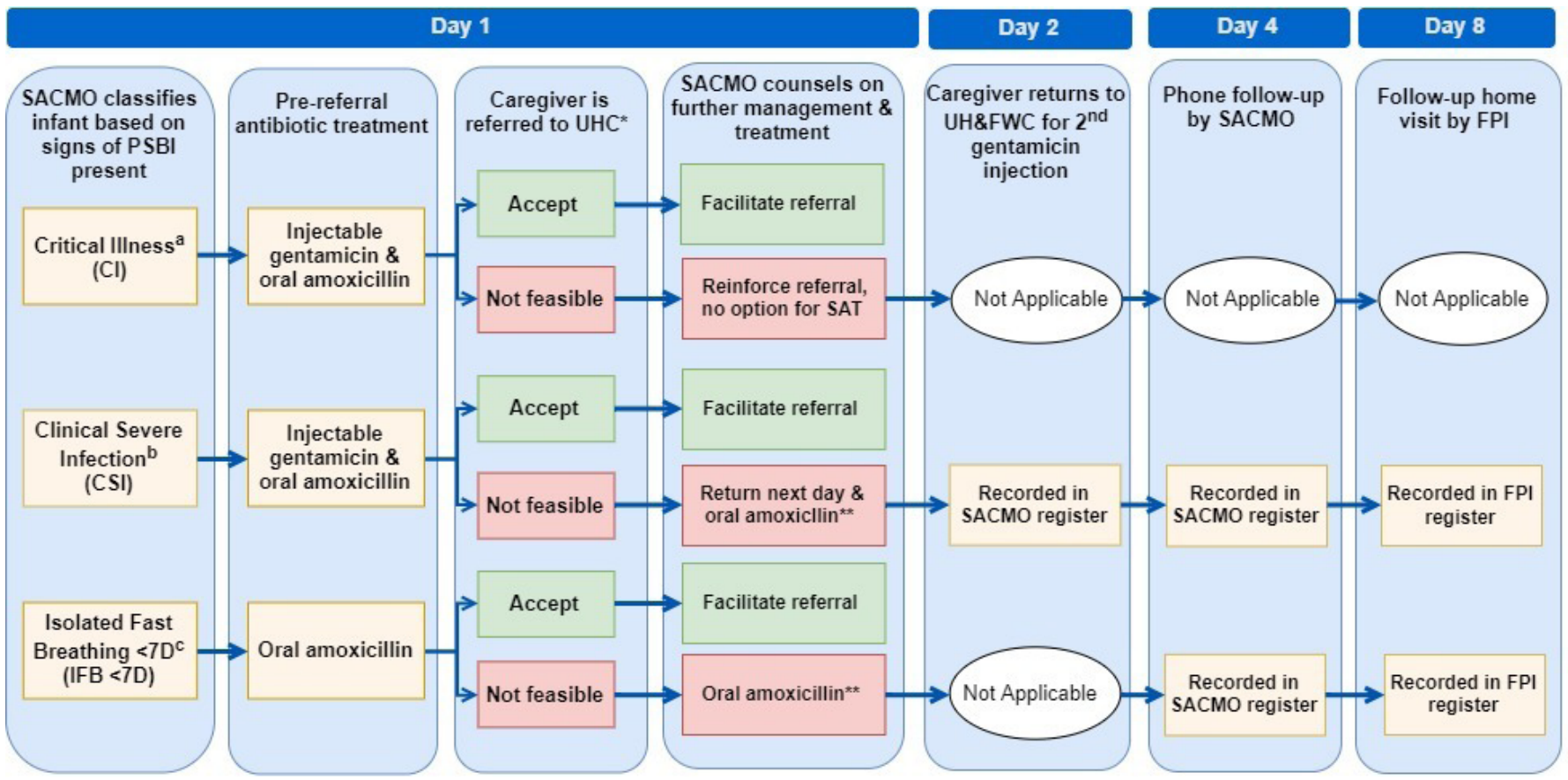

Figure 2 Management and treatment pathway for young infants with PSBI assessed and treated at UH\&FWCs according to Bangladesh's infection management guidelines. *Critical Illness signs: unconscious, convulsions or history of convulsions, unable to feed at all, no movement on stimulation, unable to cry, persistent vomiting, bulging fontanelle, cyanosis. $†$ Clinical severe infection signs: severe chest in-drawing, hypothermia $\left(<35.5^{\circ} \mathrm{C}\right)$, fever $\left(>37.5^{\circ} \mathrm{C}\right)$, movement only on stimulation, not feeding well (based on history and observation). †lsolated fast breathing ( $<7$ days): respiratory rate equal to or greater than 60 breaths per minute as the single sign of infection in infants aged $<7$ days. §UHC is the primary referral facility; however, the infant may be referred to the district hospital if it is closer to the UH\&FWC. १The SACMO counsels the caregiver on providing medicine to the infant at home and prescribes oral amoxicillin two times a day for 7 days. FPI, Family Planning Inspector; PSBI, possible serious bacterial infection; SACMO, Sub-Assistant Community Medical Officers; SAT, simplified antibiotic treatment; UHC, Upazila Health Complex; UH\&FWC, Union Health \& Family Welfare Centres. 
(CSI) or isolated fast breathing (IFB) in infants $<7$ days are eligible for simplified antibiotic treatment (SAT) - when hospital referral is not feasible for families-with injectable gentamicin once daily for 2 days and oral amoxicillin two times a day for 7 days. If referral is not feasible, caregivers of infants with CSI are instructed to return to the UH\&FWC the next day for the second gentamicin injection. The FWV may provide the second injection if the SACMO is not present at the UH\&FWC. Infants with IFB $(<7 D)$ only receive the oral amoxicillin regimen. The SACMO follows up with caregivers over telephone on the fourth day, and if the infant's condition has not improved, advises the caregiver to seek care at the UHC. On the eighth day of treatment, the family receives a home visit from the Family Planning Inspector (FPI), who are trained as supervisors of frontline workers, to assess treatment compliance and the condition of the infant. ${ }^{16}$

\section{IMPLEMENTATION STRATEGIES AND DATA COLLECTION}

In August 2015, implementation of the new guidelines started in $53 \mathrm{UH} \& \mathrm{FWCs}$ of 10 subdistricts within 3 districts of Bangladesh-Lakshmipur, Noakhali and Habiganj. We provided support to the MOHFW to ensure facility readiness, build capacity of providers and promoted awareness and community engagement with the public sector healthcare system (figure 1). Additional details on our implementation strategies are provided in online supplementary table 1 . Project staff extracted data from UH\&FWC registers maintained by the SACMO during monthly supervisory meetings at the subdistrict hospital. The information collected included age and sex of the infant, illness classification, referral decision, gentamicin treatment and follow-up. Data on oral amoxicillin treatment were not available.

Our analysis followed an explanatory sequential mixed methods design where qualitative data were analysed to help explain the quantitative results. ${ }^{29}$ We analysed data for 4597 sick young infants collected over a 15 -month period during early implementation (October 2015-December 2016). A total of 4590 records were included after excluding records missing age $(n=5)$ and illness classification $(n=2)$. Qualitative data included three focus group discussions with SACMOs $(n=2)$ and FPIs $(n=1)$ to explore providers' opinions about guideline implementation; key-informant interviews with MOHFW managers at the national and subdistrict levels and documentation of programme learnings by project staff. We used the WHO PSBI Monitoring and Evaluation Framework $^{12}$ to identify key indicators and guide our analysis of implementation research outcomes (figure 1). We performed $\chi^{2}$ tests of independence to examine the relation between PSBI classification, referral feasibility and prereferral treatment.

\section{Ensuring implementation readiness at primary healthcare facilities: feasibility of programme delivery}

We collaborated with the MOHFW to deliver project inputs and activities to 53 UH\&FWCs to ensure facility readiness for PSBI management, including training for service providers responsible for guideline implementation and provision of essential commodities (eg, gentamicin, syringes, weighing scales) and logistics (eg, registers, referral forms, job aids). Rollout of the intervention went as planned for $98 \%$ of the UH\&FWCs $(n=52 / 53)$ during the programme period. In one UH\&FWC, there was a brief period of service disruption due to the retirement of the SACMO. In response, the project worked with a neighbouring UH\&FWC to accommodate patients and fill gaps in service delivery during the interim period.

When asked about programme rollout, SACMOs reported comprehension of the guidelines and appreciation for the focused training on managing sick newborns. When probed on barriers, SACMOs reported the register is time-consuming to complete, which is challenging when the UH\&FWC is busy because they are often the only provider available to see paediatric patients. Additionally, since the PSBI management indicators are not yet included in national Management Information Systems, SACMOs are required to prepare separate monitoring reports for both PSBI management and IMCI, which they feel is a paper-based overload.

\section{Feasibility of PSBI management at UH\&FWC and guideline adherence}

MaMoni HSS provided support to MOHFW managers and providers during the programme period to promote and monitor adherence to the PSBI guidelines (figure 3 and online supplementary table 1). Our analysis of programme monitoring data included 4590 young infants, of which $514(11 \%)$ were classified as PSBI (table 1). Most infants were categorised with other illnesses $(29 \%)$ or less severe types of infection (local bacterial infection (33\%), IFB in infants 7-59 days old $(27 \%))$. Referral for hospital care, with prereferral antibiotic treatment, remains the first-line recommendation for treatment of PSBI in young infants. ${ }^{5}$ Of the 514 young infants classified with PSBI, 436 non-critically ill cases $(85 \%)$ were eligible to receive simplified treatment if referral was not feasible. Families of infants with CI had significantly higher referral acceptance $(99 \%)$ than infants with CSI $(22 \%$; $p<0.001)$ and very young infants $<7$ days with IFB $(3 \% ; \mathrm{p}<0.001)$. Regardless of referral feasibility, all CI and CSI cases should receive intramuscular gentamicin. Young infants with CSI for whom referral was not feasible had significantly higher rates of gentamicin treatment $(99 \%)$ compared with CI cases $(6 \% ; \mathrm{p}<0.001)$ and CSI cases $(52 \% ; \mathrm{p}<0.001)$ accepting referral. For CSI cases receiving outpatient treatment, $98 \%$ received the second gentamicin injection. Follow-up by the SACMO on day 4 (99\% CSI vs $6 \%$ IFB ( $<7$ days); $\mathrm{p}<0.001)$, and the FPI on day 8 ( $86 \%$ CSI vs $5 \%$ IFB ( $<7$ days); $p<0.001)$, also differed by classification.

Providers reported that families indicated economic constraints and distance to the hospital as barriers to referral feasibility. When probed about prereferral 
MaMoni HSS Support: 7-day training for SACMOS on PSBI guidelines; Joint supervision visits with MOHFW managers; Monthly meetings with SACMOS at the UHC, which served as a platform for program monitoring, monthly report preparation \& refresher sessions of technical knowledge
MaMoni HSS Support: One day orientation on guidelines for FPI $\&$ visit facilitation

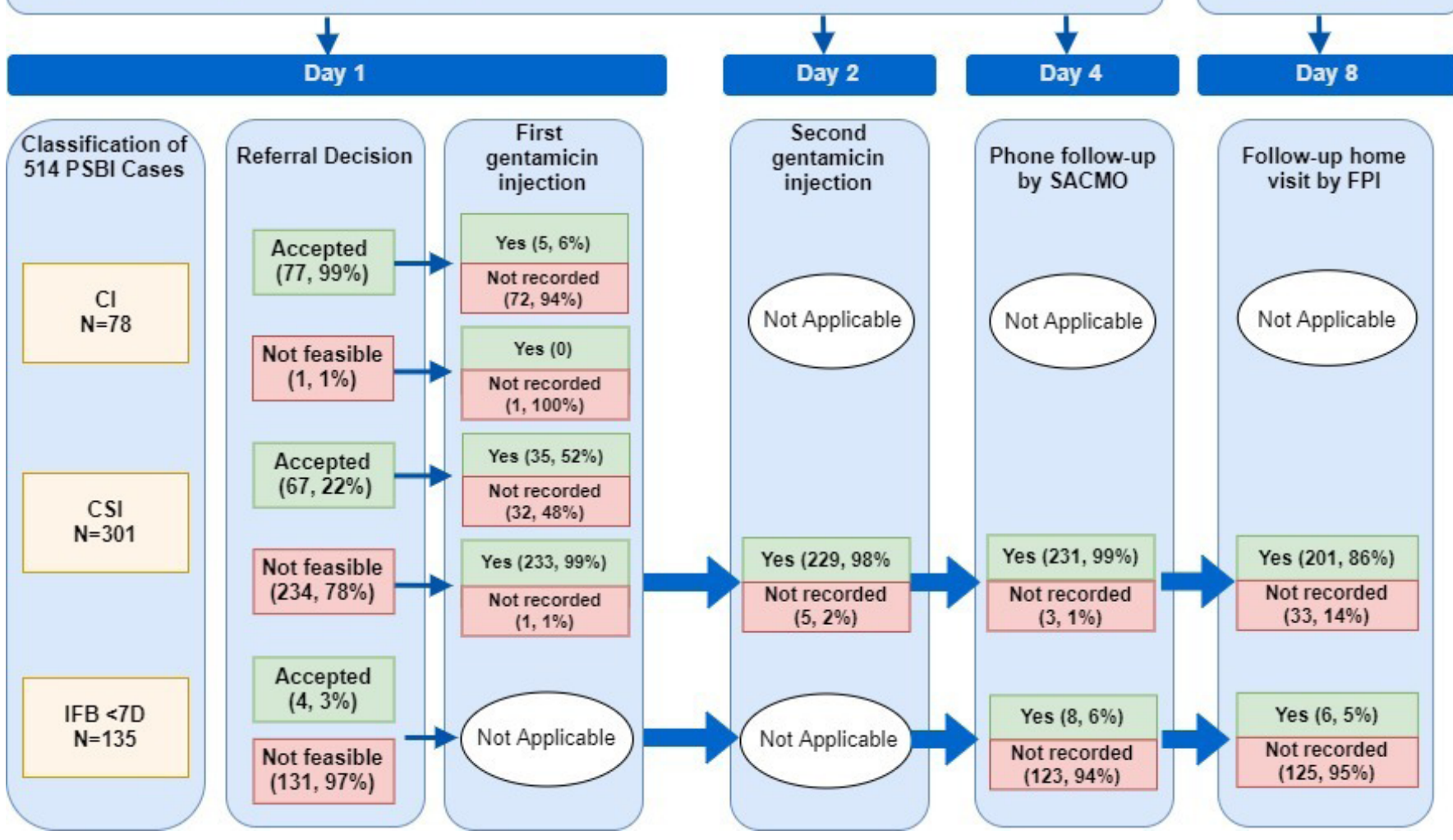

Figure 3 MaMoni HSS support to PSBI case management and programme monitoring data on referral decision, treatment and follow-up of PSBI cases (0-59 days) during the period of October 2015-December 2016. Cl, critical illness; CSI, clinical severe infection; IFB, isolated fast breathing; HSS, FPI, Family Planning Inspector; MaMoni HSS, MaMoni Health System Strengthening project; PSBI, possible serious bacterial infection; MOHFW, Ministry of Health and Family Welfare; SACMO, Sub-Assistant Community Medical Officers; UHC, Upazila Health Complex; UH\&FWC, Union Health \& Family Welfare Centres.

antibiotic treatment, providers discussed managing infants under 2 months of age is a sensitive matter. Providers are mindful that a poor outcome after treating infants with injectable antibiotics may create a negative impression in the community and expressed reservations about providing injections to very sick and very young infants. Providers did not identify challenges with day 4 follow-up but raised several challenges with the day 8 follow-up mechanism. While the national guidelines specify day 8 follow-up as the responsibility of FPIs, this is not yet formally included in their job description. FPIs also reported scheduling a home visit for the exact eighth day of treatment is challenging as it may fall on a day when they are unable to travel to the community due to other duties (eg, trainings, visits in other areas) and identified lack of transport, or reimbursement for transport costs, as barriers to completing home visits. Additionally, FPIs are meant to visit the UH\&FWC weekly to submit surveillance reports to the SACMO and identify new PSBI cases for follow-up. ${ }^{14}$ However, both FPIs and SACMOs report that these visits are not happening regularly, which may adversely affect the quality of recordkeeping and reporting.

\section{Reaching families to promote awareness and care-seeking for young infant illness}

We oriented government providers in the community and an existing pool of unpaid Community Volunteers ( 1 per 250 persons) on the guidelines to promote awareness of services and increase referrals to primary health facilities. The Community Volunteer programme model required minimal inputs from MaMoni HSS and was largely sustained by community ownership and coordination between the volunteers and government frontline workers (online supplementary table 1 ).

We estimated capture of PSBI cases, trends in utilisation and source of community referrals to assess reach and acceptability of the programme. The number of PSBI cases by month remained low, but there was an increasing trend in the total number of young infants identified at the UH\&FWC over the programme period (figure 4). The most frequently cited source of referral was a Community Volunteer $(47 \%)$ followed by selfreferrals $(36 \%)$ (table 1 ). Based on the PSBI incidence rate for young infants in South Asia (95.4/1000 live births), we estimated that $11.2 \%$ (95\% CI 10.3 to 12.1 ) of the expected PSBI cases in the programme area sought 


\begin{tabular}{|c|c|}
\hline Characteristic & $\begin{array}{l}\%(n) \\
n=4590\end{array}$ \\
\hline \multicolumn{2}{|l|}{ Age (in days) } \\
\hline$<7$ days & $10 \%(451)$ \\
\hline $7-28$ days & $41 \%(1893)$ \\
\hline 29-59 days & $49 \%(2246)$ \\
\hline \multicolumn{2}{|l|}{ Sex of infant } \\
\hline Male & $55 \%(2503)$ \\
\hline Female & $45 \%(2087)$ \\
\hline \multicolumn{2}{|c|}{ IIIness classification recorded by SACMO } \\
\hline PSBI & $11 \%(514)$ \\
\hline Critical illness & $15 \%(78)$ \\
\hline Clinical severe infection & $59 \%(301)$ \\
\hline Isolated fast breathing (<7 days) & $26 \%(135)$ \\
\hline $\begin{array}{l}\text { Isolated fast breathing (7-59 } \\
\text { days) }\end{array}$ & $27 \%(1262)$ \\
\hline Local bacterial infection & $33 \%(1500)$ \\
\hline Other illness & $29 \%(1314)$ \\
\hline \multicolumn{2}{|l|}{ Source of referral to UH\&FWC } \\
\hline Community volunteer & $47 \%(2153)$ \\
\hline Caregiver self-referred & $36 \%(1668)$ \\
\hline MOHFW provider & $9 \%(394)$ \\
\hline Informal provider & $5 \%(236)$ \\
\hline Other & $3 \%(139)$ \\
\hline
\end{tabular}

MOHFW, Bangladesh's Ministry of Health and Family Welfare; $\mathrm{PSBI}$, possible serious bacterial infection; SACMO, Sub-Assistant Community Medical Officers; UH\&FWC, Union Health \& Family Welfare Centres.

care from UH\&FWCs during this 15 -month period. ${ }^{3} 30$ Qualitative findings with providers, MOHFW managers and project staff attributed low utilisation rates to lack of awareness of services available at the UH\&FWC and caregivers' preference for private sector providers.

\section{REFLECTIONS ON PROGRAMME LEARNINGS AND RECOMMENDATIONS FOR SCALE-UP}

Ensuring facility readiness to provide PSBI services was feasible due to multistakeholder engagement and collaboration, which has been shown to be critical for implementation research and scale-up of interventions. ${ }^{17} 1831$ Barriers varied by facility depending on managing MOHFW Directorate, either DGFP or DGHS, because each has separate supply chains from the national to subdistrict levels. Most of the programme area UH\&FWCs are under administrative management of DGFP, which did not have supply of injectable gentamicin required for PSBI management in the local supply chain. While DGHS had injectable gentamicin available, there was not an adequate supply available for distribution to the UH\&FWCs. To support plans for scale-up, we collaborated with stakeholders, including MOHFW managers in both Directorates, to identify bottlenecks and plan for the necessary financing in absence of project support. As a result, in the current health sector plan, both Directorates have secured the necessary budget for the procurement of essential commodities for PSBI management in UH\&FWCs, incorporated plans for capacity building and allocated resources for training of providers in PSBI management guidelines. ${ }^{14}$ Following global guidance, the government has also undertaken initiatives to align the PSBI guidelines with the existing IMCI programme, including reporting through District Health Information System 2 for those UH\&FWCs that are managed by DGHS. ${ }^{12} 13$ A similar electronic information system is under development, with support from MaMoni HSS, for UH\&FWCs managed by DGFP. Both electronic systems present an opportunity for reducing the recordkeeping burden for SACMOs, improving monitoring practices and quality of reporting.

Provider adherence to the guidelines varied by infection classification and referral feasibility. Substantial time in training and supervision focused on management of CSI cases when referral was not feasible because these infants require two gentamicin injections and follow-up on the fourth and eighth day. Therefore, providers may have prioritised the outpatient management of CSI cases, indicated by the high level of adherence to treatment and follow-up of these cases. Prereferral antibiotic treatment was significantly lower for cases that accepted referral. From a clinical perspective, referral without prereferral antibiotics is a life-threatening lapse in care. Newborns with severe infections can deteriorate rapidly if left untreated; thus, timely treatment with antibiotics is critical. ${ }^{32}{ }^{33}$ Provider reservations about providing injections to critically ill infants may contribute to this lapse in care. Strategies to improve providers' confidence to provide prereferral injections, and emphasis on follow-up of all PSBI cases, should be included in future training and supervision sessions.

Uncertainty around treatment outcomes, especially for referred cases, is a major barrier to monitoring the long-term effectiveness of the programme. The guidelines recommend that SACMOs follow-up with the caregiver, or hospital providers, to ensure families reach the referral facility; however, this follow-up is not recorded in the registers, so the health outcomes of these infants are not routinely tracked. Families that accept referral are more likely to have infants with serious illness and in the current system are more likely to be lost to follow-up. Interventions to mitigate the financial burden of hospital referral, including transfer arrangements, could improve feasibility of referral for families. Systems for tracking referral cases need to be established and tested during rollout of the guidelines, and indicators for monitoring the care and outcomes of these cases should be integrated into routine systems. For PSBI cases receiving outpatient treatment, the day 8 follow-up visit is critical for assessing 


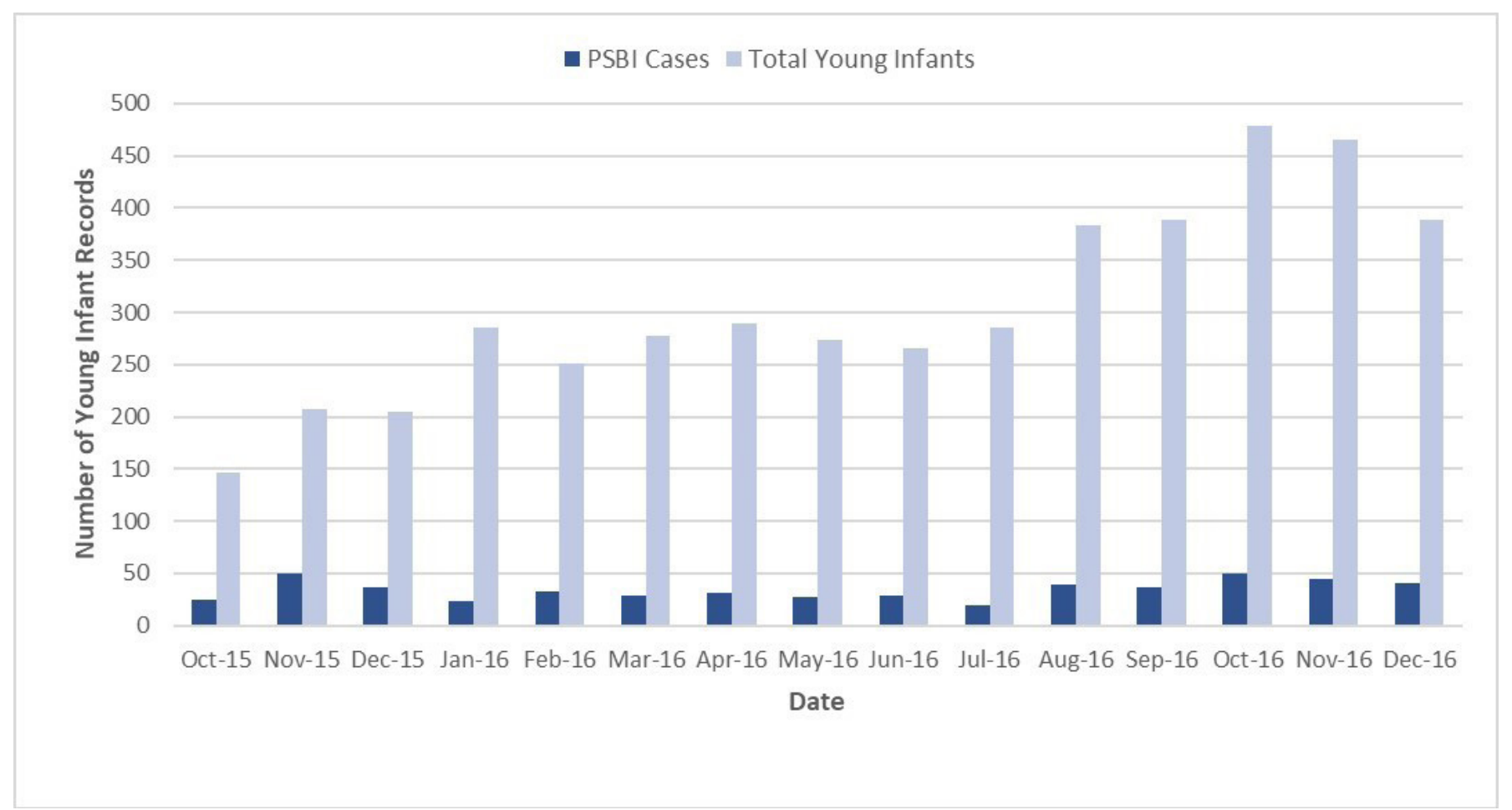

Figure 4 Utilisation of the UH\&FWC for young infants and PSBI cases identified from October 2015 to December 2016. PSBI, possible serious bacterial infection; UH\&FWC, Union Health \& Family Welfare Centres.

treatment outcomes. However, we found this visit was not regularly happening. We responded to this barrier by facilitating follow-up during the programme period, which allowed for these visits to occur, but limited our ability to assess the functionality of these visits in the routine system. This illustrates a common trade-off with implementation research, which others have suggested could be balanced by studying interventions within the context of multiple settings. ${ }^{17} 34$ Further investigation into improving functionality of these visits is needed as this strategy is scaled-up in Bangladesh and within multicountry contexts to improve external validity.

Since our data are from early implementation, we anticipated low rates of capture of PSBI cases at UH\&FWCs. Qualitative findings attributed low utilisation to caregivers' preference for private sector providers and lack of awareness of services available at the UH\&FWC. Bangladesh's private sector offers high-end services for wealthy families, while a large economy of informal providers retails services in the community. ${ }^{19}$ Previous studies on care-seeking for neonatal illness in Bangladesh have identified a preference for informal providers as families may perceive their practices as more convenient, affordable or culturally appropriate ${ }^{19}{ }^{35-37}$ In the early stages of implementation, it is important to focus on number of cases identified and trends in utilisation. ${ }^{12}$ Community Volunteers were frequently cited as a source of referral, which indicates penetration of our community engagement programme. Increasing trends in utilisation suggest improved awareness and acceptability of UH\&FWC services among families of young infants as the programme matured, which over time, may contribute to a higher capture of PSBI cases in the community.

Through our reflection on programme leanings, we identified some important limitations in our study data. Programme monitoring was based on facility records and interviews with providers, which limited our ability to investigate barriers to programme feasibility and acceptability from the caregivers' perspective. Future studies should include interviews with caregivers to collect data on predictors of newborn infections, explore the complexities around referral feasibility and adapt strategies to improve access to and acceptability of services. Missing data in facility records limited our ability to draw conclusions about the effectiveness of simplified treatment and compare treatment outcomes across subcategories. Indicators on treatment and follow-up of cases were especially vulnerable to this limitation because it was not possible to determine in the analysis phase if blank fields indicated that treatment/follow-up was not completed, or if the fields were blank due to recording errors. There is variation among providers in how they record and report data, which was not controlled for in our data collection. Therefore, our data are subject to reporting bias and performance on care indicators should be interpreted judiciously. Future programme efforts should review register completeness during supervision, or periodic programme review, to promote accountability and address challenges associated with incomplete registers in a timely manner. 


\section{CONCLUSION}

We set out to improve the feasibility of programme delivery, including promoting facility readiness and provider adherence to the guidelines, through embedding implementation strategies in the local context. Bangladesh's current health sector plan has allocated budget for training of providers, procurement of drugs and equipment and improved availability of PSBI services, thereby enhancing the feasibility of scale-up. ${ }^{14}$ As this intervention is scaled up, it is imperative to ensure high quality care at UH\&FWCs and referral facilities for all PSBI cases. High provider adherence to the guidelines for CSI cases suggests outpatient treatment of PSBI is feasible, but performance was not uniform across infection classifications. Periodic observation and assessments of service delivery, for both SACMOs and FPIs, should be included in supervision to identify context-specific challenges and develop strategies for improving prereferral antibiotic treatment and follow-up of PSBI cases. Complete treatment data, including indicators on oral amoxicillin treatment, are needed to better assess the coverage and effectiveness of SAT. Systems for tracking of referral cases are needed to ensure families reach higherlevel facilities and monitor treatment outcomes. This will require coordination within the MOHFW to strengthen systems for communication between providers at the union and subdistrict levels. The MOHFW's current efforts to align PSBI management with IMCI provides an opportunity for harmonising implementation with the ongoing IMCI programme, and expansion of digital information systems offer opportunities to incorporate referral follow-up in monitoring and reporting requirements.

Our community engagement programme used an existing pool of Community Volunteers who worked as an extended arm of the government frontline workers to promote awareness and appropriate care-seeking. Few young infants with PSBI sought care from UH\&FWCs in the programme area during early implementation. However, changing care-seeking behaviour takes time. Our community engagement programme helped to reach caregivers and contributed to an increasing trend in utilisation of UH\&FWCs. Future programme activities should include interviews with caregivers to explore the complexities around referral feasibility, acceptability of SAT and adapt strategies to respond to the needs of families within the public sector health system.

\author{
Author affiliations \\ ${ }^{1}$ International Health, Johns Hopkins University Bloomberg School of Public Health, \\ Baltimore, Maryland, USA \\ ${ }^{2}$ MaMoni Health Systems Strengthening Project, Save the Children, Dhaka, Dhaka \\ District, Bangladesh \\ ${ }^{3}$ National Newborn Health Program, Government of Bangladesh Ministry of Health \\ and Family Welfare, Dhaka, Dhaka District, Bangladesh \\ ${ }^{4}$ United States Agency for International Development, Dhaka, Bangladesh \\ ${ }^{5}$ Save the Children, Washington, District of Columbia, USA
}

Acknowledgements We would like to acknowledge MOHFW service providers at the frontline, supervisors and managers who took time out of their busy schedules to discuss their opinions about implementation of the PSBI guidelines with us. We would also like to acknowledge the Community Volunteers that supported the government frontline community workers and contributed data from their records to this analysis. We are grateful to USAID for providing financial and technical inputs to this project and our technical advisors at Save the Children and the Johns Hopkins Bloomberg School of Public Health. Finally, we would like to thank all the MaMoni HSS staff and partners who supported MOHFW implementation in the field, without which this paper would not be possible.

Contributors JG led the MaMoni HSS project in Bangladesh with technical support from SabA, MAK, JI, MB, IR and SanA to implement program activities and collect data. JG, JAA, IR and SabA conceptualised the manuscript. JA, SabA, SanA, MI and MAK generated the qualitative reports. JAA conducted the analysis, created the tables and figures, was the principal author and managed all revisions of the drafts of the paper. NK, SanA and SabA managed the program database and assisted with data analysis. JG and $A B$ provided guidance to the analysis and drafts of the paper. JJ, SW, IR and JI provided comments on the intellectual content for the paper. All authors approved the final draft of the manuscript.

Funding This manuscript is made possible by the support of the American people through the United States Agency for International Development (USAID); Associate Cooperative Agreement no. AID-338-LA-13-00004 - MaMoni Health Systems Strengthening (MaMoni HSS). The contents are the sole responsibility of USAID's MaMoni HSS Project and do not necessarily reflect the views of USAID or the United States Government.

Disclaimer The contents are the sole responsibility of USAID's MaMoni HSS Project and do not necessarily reflect the views of USAID or the US Government.

Competing interests None declared.

Patient consent for publication Not required.

Ethics approval The data presented in this paper are from program monitoring and were used by the MaMoni HSS team for quality improvement efforts. At the time of manuscript development, this project was reviewed by the Johns Hopkins School of Public Health (JHSPH) Institutional Review Board who granted a waiver and made the determination that JHSPH and its faculty, staff and students are 'not engaged in human subjects research' for this project.

Provenance and peer review Not commissioned; externally peer reviewed. Data availability statement № additional data are available.

Open access This is an open access article distributed in accordance with the Creative Commons Attribution Non Commercial (CC BY-NC 4.0) license, which permits others to distribute, remix, adapt, build upon this work non-commercially, and license their derivative works on different terms, provided the original work is properly cited, appropriate credit is given, any changes made indicated, and the use is non-commercial. See: http://creativecommons.org/licenses/by-nc/4.0/.

\section{ORCID iDs}

Jennifer A Applegate http://orcid.org/0000-0002-9472-299X

Iftekhar Rashid http://orcid.org/0000-0003-2775-3435

\section{REFERENCES}

1 You D, Hug L, Ejdemyr S. Levels and trends in child mortality. Report 2015. UN Inter-agency Group for Child Mortality Estimation, 2015.

2 Liu L, Oza S, Hogan D, et al. Global, regional, and national causes of under-5 mortality in 2000-15: an updated systematic analysis with implications for the sustainable development goals. Lancet 2016;388:3027-35

3 National Institute of Population Research and Training (NIPORT), Mitra and Associates, and ICF International. Bangladesh demographic and health survey 2014. Dhaka, Bangladesh, and Rockville, Maryland, USA: NIPORT, Mitra and Associates, and ICF International, 2016.

4 Ahmed I, Ali SM, Amenga-Etego S, et al. Population-Based rates, timing, and causes of maternal deaths, stillbirths, and neonatal deaths in South Asia and sub-Saharan Africa: a multi-country prospective cohort study. Lancet Glob Health 2018;6:e1297-308.

5 World Health Organization. Guideline: managing possible serious bacterial infection in young infants when referral is not feasible. Geneva, Switzerland: WHO Press, World Health Organization, 2015.

6 Baqui AH, Saha SK, Ahmed ASMNU, et al. Safety and efficacy of alternative antibiotic regimens compared with 7 day injectable procaine benzylpenicillin and gentamicin for outpatient treatment of neonates and young infants with clinical signs of severe infection 
when referral is not possible: a randomised, open-label, equivalence trial. Lancet Glob Health 2015;3:e279-87.

7 Mir F, Nisar I, Tikmani SS, et al. Simplified antibiotic regimens for treatment of clinical severe infection in the outpatient setting when referral is not possible for young infants in Pakistan (Simplified Antibiotic Therapy Trial [SATT]): a randomised, open-label, equivalence trial. Lancet Glob Health 2017;5:e177-85.

8 Tshefu A, Lokangaka A, Ngaima S, et al. Simplified antibiotic regimens compared with injectable procaine benzylpenicillin plus gentamicin for treatment of neonates and young infants with clinical signs of possible serious bacterial infection when referral is not possible: a randomised, open-label, equivalence trial. The Lancet 2015;385:1767-76.

9 Esamai F, Tshefu AK, Ayede Al, et al. Ongoing trials of simplified antibiotic regimens for the treatment of serious infections in young infants in South Asia and sub-Saharan Africa: implications for policy. Pediatr Infect Dis J 2013;32(Suppl 1):S46-9.

10 Qazi SA, Wall S, Brandes N, et al. An innovative multipartner research program to address detection, assessment and treatment of neonatal infections in low-resource settings. Pediatr Infect Dis $J$ 2013;32(Suppl 1):S3-6.

11 Theobald S, Brandes N, Gyapong M, et al. Implementation research: new imperatives and opportunities in global health. Lancet 2018;392:2214-28.

12 World Health Organization. Operationalizing management of sick young infants with possible serious bacterial infection ( $P s b l)$ when referral is not feasible in the context of existing maternal, newborn, and child health programmes. Geneva: World Health Organization and the United Nations Children's Fund (UNICEF), 2017.

13 World Health Organization, UNICEF. Managing possible serious bacterial infection in young infants $0-59$ days old when referral is not feasible: WHO/UNICEF joint statement. World Health Organization, 2017.

14 Bangladesh Ministry of Health and Family Welfare. National newborn health program (NNHP). Dhaka, Bangladesh, 2017.

15 Government of Bangladesh. End preventable child deaths by 2035: Bangladesh call for action; 2013.

16 National Technical Working Committee on Newborn Health. Bangladesh national guideline: management of infection of the 0-59 days infants at Union level facilities and NGO clinics without indoor facilities; 2015

17 Alonge O, Rodriguez DC, Brandes N, et al. How is implementation research applied to advance health in low-income and middleincome countries? BMJ Glob Health 2019;4:e001257.

18 Peters DH, Adam T, Alonge O, et al. Implementation research: what it is and how to do it. BMJ 2013;347:f6753.

19 Ahmed SM, Evans TG, Standing H, et al. Harnessing pluralism for better health in Bangladesh. Lancet 2013;382:1746-55.

20 Asia Pacific Observatory on health systems and policies. Bangladesh health system in review. Health systems in transition. 5. World Health Organization, 2015

21 Khan M, Horchkiss D. Bangladesh health facility survey 2009. Dhaka: World Bank, 2010.
22 Arifeen SE, Bryce J, Gouws E, et al. Quality of care for under-fives in first-level health facilities in one district of Bangladesh. Bull World Health Organ 2005;83:260-7.

23 Chowdhury EK, El Arifeen S, Rahman M, et al. Care at first-level facilities for children with severe pneumonia in Bangladesh: a cohort study. The Lancet 2008;372:822-30.

24 Chowdhury S, Hossain SA, Halim A. Assessment of quality of care in maternal and newborn health services available in public health care facilities in Bangladesh. Bangladesh Med Res Counc Bull 2009;35:53-6.

25 Arifeen SE, Hoque DME, Akter T, et al. Effect of the integrated management of childhood illness strategy on childhood mortality and nutrition in a rural area in Bangladesh: a cluster randomised trial. Lancet 2009;374:393-403.

26 Rahman AE, lqbal A, Hoque DME, et al. Managing neonatal and early childhood syndromic sepsis in Sub-District hospitals in resource poor settings: improvement in quality of care through introduction of a package of interventions in rural Bangladesh. PLoS One 2017;12:e0170267.

27 Rowe AK, Labadie G, Jackson D, et al. Improving health worker performance: an ongoing challenge for meeting the sustainable development goals. BMJ 2018;362.

28 Siron S, Dagenais C, Ridde V. What research tells us about knowledge transfer strategies to improve public health in low-income countries: a scoping review. Int $J$ Public Health 2015;60:849-63.

29 Creswell J, Clark VP. Designing and conducting mixed methods research. 2nd edn. USA: SAGE Publication Inc, 2011.

30 Saha SK, Schrag SJ, El Arifeen S, et al. Causes and incidence of community-acquired serious infections among young children in South Asia (anisa): an observational cohort study. Lancet 2018;392:145-59.

31 Keene CM, Aluvaala J, Murphy GAV, et al. Developing recommendations for neonatal inpatient care service categories: reflections from the research, policy and practice interface in Kenya. BMJ Glob Health 2019;4:e001195.

32 Edmond K, Zaidi A. New approaches to preventing, diagnosing, and treating neonatal sepsis. PLoS Med 2010;7:e1000213.

33 Simen-Kapeu A, Seale AC, Wall S, et al. Treatment of neonatal infections: a multi-country analysis of health system bottlenecks and potential solutions. BMC Pregnancy Childbirth 2015;15(Suppl 2):S6.

34 Green LW, Nasser M. Furthering dissemination and implementation research. In: Dissemination and implementation research in health: translating science to practice. 301. Oxford Scholarship Online, 2017.

35 Chowdhury SK, Billah SM, Arifeen SE, et al. Care-seeking practices for sick neonates: findings from cross-sectional survey in 14 rural sub-districts of Bangladesh. PLoS One 2018;13:e0204902.

36 Darmstadt GL, Syed U, Patel Z, et al. Review of domiciliary newborn-care practices in Bangladesh. J Health Popul Nutr 2006;24:380.

37 Shah R, Mullany LC, Darmstadt GL, et al. Determinants and pattern of care seeking for preterm newborns in a rural Bangladeshi cohort. BMC Health Serv Res 2014;14:417. 